\title{
COOPERATIVE COMMUNICATION WITH SCHEME SELECTION
}

\author{
Seyed Vahid Mortezavi Zadeh', Bmehdi Fartash ${ }^{2}$
}

1 Department of Computer Engineering, Islamic Azad University, Arak Branch, Arak, Iran, e-mail: m-fartash@ iau-arak.ac.ir

Received: 2016.04.15

Accepted: 2016.05.10

Published: 2016.06.01

\begin{abstract}
In this paper, a new cooperation scheme is provided that implements the advantages of both DF and AF schemes to enhance the performance in wireless networks. We suppose that nodes can access the channel information and based on this, the cooperation scheme is selected and applied. This algorithm is repeated when channel states have an effective variety. We apply this strategy to a single relay system with square MQAM signals where optimum power allocation is employed. Simulations and comparisons verify that symbol-error-rate (SER) performance can be improved properly.
\end{abstract}

Keywords: cooperative communication, channel information, symbol error rate, amplify and forward, decode and forward.

\section{INTRODUCTION}

Recently, cooperative communications has been proposed for wireless networks [1-7]. The basic idea is that in addition to the direct transmission, there may be other nodes that can relay the source information to the destination and diversity is enhanced. Various practical schemes have been proposed to exploit the benefits of cooperation among nodes. These schemes are usually classified into two categories: amplifyand-forward (AF) and decode-and-forward (DF) [1]. In AF, a relay node simply amplifies and retransmits the signal waveform received from a source. In DF, the received signal in relay node is demodulated and decoded before transmission. Due to the possibility of decoding error, DF can stop the cooperation scenario when this error probability is high. This can be determined by several methods like the source to relay channel quality information that is provided to nodes. Each scenario has its benefits and we can choose a cooperation scheme that is suitable for network conditions. AF requires low implementation complexity at the relay, and operates under all source-relay (S-R) channel conditions. How- ever, AF also amplifies the noise power at the same time. Otherwise, DF can achieve a higher quality and performance when the S-R channel is good and the signal can be decoded correctly at the relay node $[5,8]$.

In [9], the symbol-error-rate (SER) performance of uncoded cooperation systems with either DF or AF cooperation protocols was investigated. Since the closed-form SER formulation is complicated, its asymptotically tight approximations at high signal-to-noise ratios (SNR) with PSK and square QAM constellations were derived. Based on these analyses, an asymptotically optimum power allocation procedure has been proposed.

In this paper, a simple relay channel is considered as shown in Figure 1 in which the channel coefficients are assumed to be circularly symmetric Gaussian distributed, and independent from each other. Furthermore, square MQAM constellations are used in nodes and power allocation scheme is optimum [9]. Based on channel information, relay node selects the appropriate cooperation scheme and send its decision to destination. This algorithm is repeated when information of channels have an effective variety. 


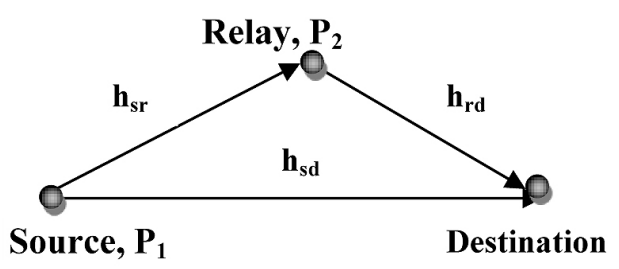

Fig. 1. A simplified cooperation model

\section{SYSTEM MODEL}

We consider a cooperation strategy with two Phases. In Phase 1, the source broadcasts its data to the desired destination, and other neighboring nodes simultaneously. In Phase 2, the neighbors help the source by forwarding the information received in Phase 1. They may decode the received data and forward it, or amplify and forward it. We suppose that in both phases, all users transmit signals through orthogonal channels using TDMA, FDMA or CDMA schemes. For simplicity, we focus on a two-user cooperation scheme called relay channel.

In Phase 1, the source broadcasts its data to both the destination and the relay. The received signals y_sd and y_sr at the destination and the relay are, respectively:

$$
\begin{aligned}
& y_{s d}=\sqrt{\frac{P_{1}}{P_{a v}}}\left(x_{1}+\mathrm{j} x_{2}\right)\left|h_{s d}\right| e^{\mathrm{j} \nless h_{s d}}+\left|n_{s d}\right| e^{\mathrm{j} \nless n_{s d}} \\
& y_{s r}=\sqrt{\frac{P_{1}}{P_{a v}}}\left(x_{1}+\mathrm{j} x_{2}\right)\left|h_{s r}\right| e^{\mathrm{j} \nless h_{s r}}+\left|n_{s r}\right| e^{\mathrm{j} \nless n_{s r}}
\end{aligned}
$$

in which $\mathrm{P}_{1}$ is the transmitted power from the source, $x=\left(x_{1}+j x_{2}\right)$ is the transmitted data symbol with average energy $\mathrm{P}_{\mathrm{av}}[10], \mathrm{n}_{\mathrm{sd}}$ and $\mathrm{n}_{\mathrm{sr}}$ are additive noises, and $h_{s d}$ and $h_{s r}$ are the channel coefficients from the source to destination and relay, respectively. They are modeled as zero-mean, complex Gaussian random variables with variances $\sigma_{\mathrm{sd}}^{2}$ and $\sigma_{\mathrm{sr}}^{2}$ respectively. The noise terms $\mathrm{n}_{\mathrm{sd}}$ and $\mathrm{n}_{\mathrm{sr}}$ are also zero-mean complex Gaussian random variables with common variance $\mathrm{N}_{0}$. In Phase 2, with DF protocol, the relay forwards the correctly decoded symbol with power $\mathrm{P}_{2}$ to the destination with a similar modulation type, or stays idle otherwise. The received signal at the destination in Phase 2 becomes :

$y_{r d}=\sqrt{\frac{\tilde{P}_{2}}{P_{a v}}}\left(x_{1}+\mathrm{j} x_{2}\right)\left|h_{r d}\right| e^{\mathrm{j} \nless h_{r d}}+\left|n_{r d}\right| e^{\mathrm{j} \nless n_{r d}}$ where $\tilde{\mathrm{P}_{2}}=\mathrm{P}_{2}$ if the relay decodes the transmitted symbol correctly, and $\mathrm{P}_{2}=0$ otherwise. In (3), $h_{r d}$ is the channel coefficient from the relay to the destination, and it is modeled by a zeromean, complex Gaussian random variable with variance $\sigma_{\mathrm{rd}}^{2}$. The noise term $\mathrm{n}_{\mathrm{rd}}$ is also assumed a zero-mean complex Gaussian random variable with variance $\mathrm{N}_{0}$. In practice, we may apply an SNR threshold at the relay. If the received SNR is higher than the threshold, then the symbol is correctly decoded with a high probability. Details of threshold optimization at the relay can be found in [11].

With AF scenario, the relay amplifies the received signal and forwards it to the destination with transmitted power $\mathrm{P}_{2}$ in Phase 2. The received signal at the destination in this Phase is specified by:

$y_{r d}=\sqrt{\frac{P_{2}}{P_{1}\left|h_{s r}\right|^{2}+N_{0}}}\left(y_{s r}\right)\left|h_{r d}\right| e^{\mathrm{j} \nless h_{r d}}+\left|n_{r d}\right| e^{\mathrm{j} \nless n_{r d}}$

where $h_{r d}$ is the channel coefficient from the relay to the destination and $\mathrm{n}_{\mathrm{rd}}$ is an additive noise, with the same statistical models as in (3). More explicitly $\mathrm{y}_{\mathrm{rd}}$ :

$$
\begin{gathered}
y_{r d}=\sqrt{\frac{P_{1} P_{2}}{P_{a v}\left(P_{1}\left|h_{s r}\right|^{2}+N_{0}\right)}}\left|h_{s r}\right|\left|h_{r d}\right| e^{\mathrm{j}\left(\star h_{r d}+\nless h_{s r}\right)}\left(x_{1}+\mathrm{j} x_{2}\right) \\
+\left|n_{r d}^{\prime}\right| e^{\mathrm{j} \nless r_{r d}^{\prime}}
\end{gathered}
$$

where:

$\mathrm{n}_{\mathrm{rd}}^{\prime}=\sqrt{\frac{\mathrm{P}_{2}}{\mathrm{P}_{1}\left|\mathrm{~h}_{\mathrm{sr}}\right|^{2}+\mathrm{N}_{0}}}\left|\mathrm{n}_{\mathrm{sr}}\right|\left|\mathrm{h}_{\mathrm{rd}}\right| \mathrm{e}^{\mathrm{j}\left(\nless \mathrm{h}_{\mathrm{rd}}+\nless \mathrm{n}_{\mathrm{sr}}\right)}+\left|\mathrm{n}_{\mathrm{rd}}\right| \mathrm{e}^{\mathrm{j} \nless \mathrm{n}_{\mathrm{rd}}}(6)$

Under the assumption that $\mathrm{n}_{\mathrm{sr}}$ and $\mathrm{n}_{\mathrm{rd}}$ are independent, the equivalent noise component, $\mathrm{n}_{\mathrm{rd}}$, is a zero-mean complex Gaussian random variable with variance: $\left(1+\frac{\mathrm{P}_{2}\left|\mathrm{~h}_{\mathrm{rd}}\right|^{2}}{\mathrm{P}_{1}\left|\mathrm{~h}_{\mathrm{sr}}\right|^{2}+\mathrm{N}_{0}}\right) \mathrm{N}_{0}[10]$.

In both the DF and AF cooperation protocols, the channel coefficients $h_{s d}, h_{s r}$ and $h_{r d}$ are assumed independent and the mobility and positioning of the nodes are incorporated into the channel statistical model. The channel coefficients are assumed to be estimated at the receiver, not at the transmitter.

The destination combines the received signals from the source in Phase 1 and relay in Phase 2, and extracts the transmitted symbols using maximal-ratio combining (MRC) procedure [12]. In both protocols we fix the total transmitted power $\mathrm{P}$ :

$$
\mathrm{P}_{1}+\mathrm{P}_{2}=\mathrm{P}
$$



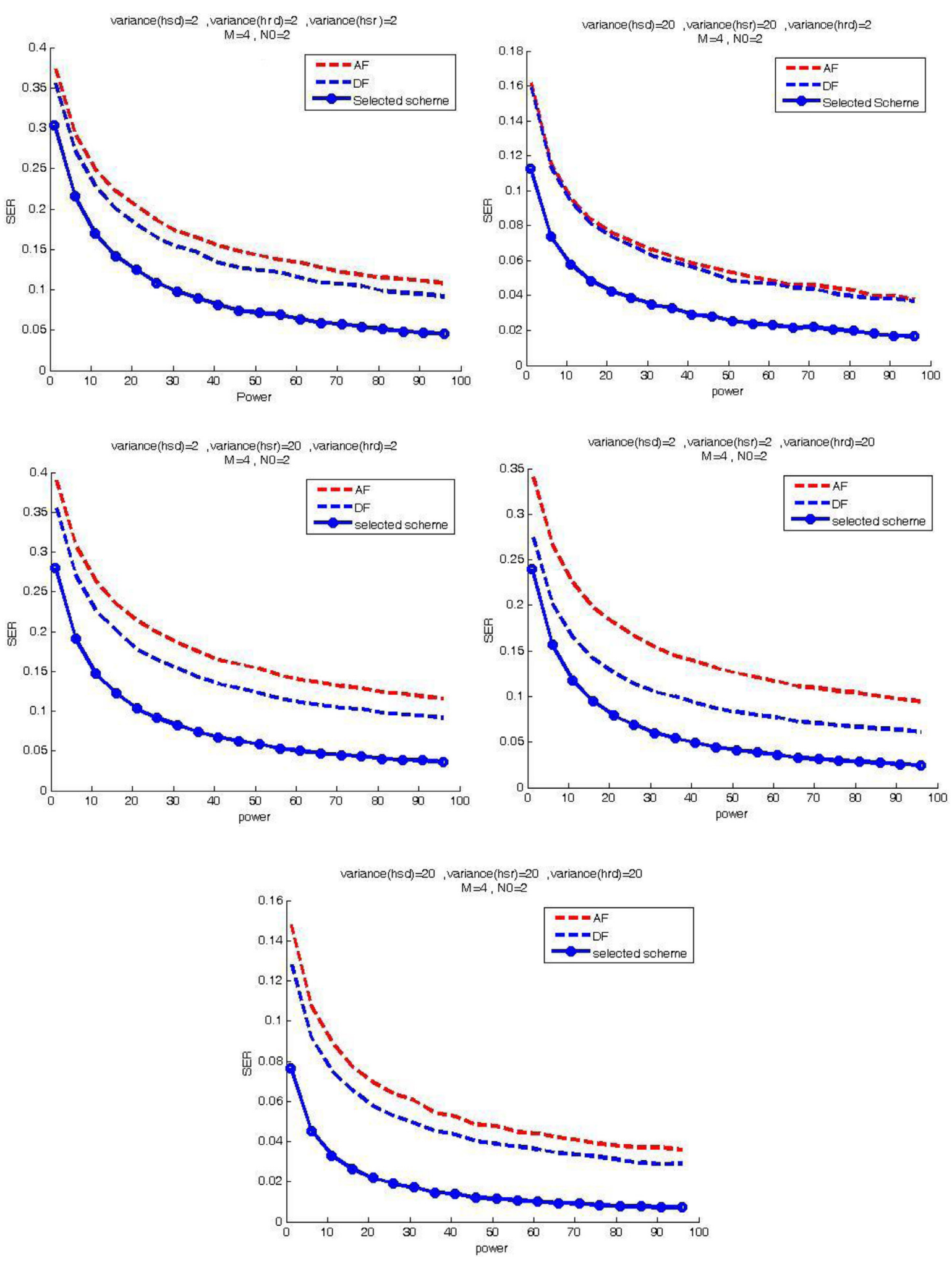

Fig. 2. Comparison of the proposed scheme and pure AF and DF cooperation scenario with 4-QAM signaling

\section{COOPERATIVE SCHEME SELECTION}

In this section, we propose a new cooperation strategy to reduce SER. With this assumption that nodes can access to the channel information, relay can gain the conditional symbol error probability of both DF and AF schemes.

With the knowledge of the channel coefficients $h_{s d}$ and $h_{r d}$, the destination extracts the transmitted symbols by combining $\mathrm{y}_{\mathrm{sd}}$ from the 
source and $\mathrm{y}_{\mathrm{rd}}$ from the relay with MRC receiver. In square M-QAM constellations, the minimum achievable conditional SER (at destination) can be expressed as:

$$
\begin{gathered}
\mathrm{p}_{\mathrm{s}, \mathrm{DF} \mid \mathbb{\mathrm { P }}_{2}=\mathrm{P}_{2}}^{\mathrm{h}_{\mathrm{sd}} \mathrm{h}_{\mathrm{r}} \mathrm{h}_{\mathrm{rd}}}=4 \mathrm{KQ}\left(\sqrt{\frac{2\left(\gamma_{1, \mathrm{DF}}+\gamma_{2, \mathrm{DF}}\right)}{\mathrm{P}_{\mathrm{av}}}}\right) \\
-4 \mathrm{~K}^{2} \mathrm{Q}^{2}\left(\sqrt{\frac{2\left(\gamma_{1, \mathrm{DF}}+\gamma_{2, \mathrm{DF}}\right)}{\mathrm{P}_{\mathrm{av}}}}\right) \\
\mathrm{p}_{\mathrm{s}, \mathrm{DF} \mid \widetilde{\mathrm{P}}_{2}=0}^{\mathrm{h}_{\mathrm{sd}}}=4 \mathrm{KQ}\left(\sqrt{\frac{2 \gamma_{1, \mathrm{DF}}}{\mathrm{P}_{\mathrm{av}}}}\right) \\
-4 \mathrm{~K}^{2} \mathrm{Q}^{2}\left(\sqrt{\frac{2 \gamma_{1, \mathrm{DF}}}{\mathrm{P}_{\mathrm{av}}}}\right) \\
\mathrm{p}_{\mathrm{s}, \mathrm{AF}}^{\mathrm{h}_{\mathrm{sd}}, \mathrm{h}_{\mathrm{sr}}, \mathrm{h}_{\mathrm{rd}}}=4 \mathrm{KQ}\left(\sqrt{\frac{2 \gamma_{\mathrm{AF}}}{\mathrm{P}_{\mathrm{av}}}}\right) \\
-4 \mathrm{~K}^{2} \mathrm{Q}^{2}\left(\sqrt{\frac{2 \gamma_{\mathrm{AF}}}{\mathrm{P}_{\mathrm{av}}}}\right)
\end{gathered}
$$

in which $\mathrm{K}=1-\frac{1}{\sqrt{\mathrm{M}}}$ and $\mathrm{Q}(\mathrm{u})=\int_{\mathrm{u}}^{\infty} \exp \left(-\frac{\mathrm{t}^{2}}{2}\right) \mathrm{dt}$ is Marcum Q-function [13-14]. $\gamma_{1, \mathrm{DF}}$ and $\gamma_{2, \mathrm{DF}}$ represent the SNR values of the first and second phase of DF scenario and $\gamma_{\mathrm{AF}}$ is the SNR value of AF cooperation strategy all are measured in the input of the receiver. Furthermore we apply the optimum power allocation schemes to achieve the optimum SER.

Based on this result, relay implements one that less conditional symbol error probability belongs it. This strategy increase the CPU overloads. In some situations performance enhancement is more significant than CPU overloads or we can handle this overload. So we choose this scheme and apply it.

Furthermore we can apply SER and its tight approximations of it instead of conditional symbol error probability to reduce the calculations of this strategy. Studies in [14] and [10] suggest good and tight approximations of SER when we access to perfect and imperfect channel information respectively.

We should notice that relay in each transmission must send destination node that what strategy is selected. We can perform this with one control bit from relay to destination. This can transmit within data frame or carry along a secure channel.

\section{SIMULATION RESULTS}

We illustrate the performance of the proposed selected scheme algorithm through computer simulations. In all simulations, we assumed a cooperation system with the variance of the noise is 1 (i.e., $\mathrm{N}_{0}=2$ ). Without less of generality in all simulation we consider a cooperation system with 4-QAM signals.

At first, we compare the resultant SER of the proposed scheme with pure DF and AF strategy. From Figure 2, we observe that the performance of this selection scheme is improved with optimum power allocation in different channel qualities.

\section{CONCLUSION}

In this paper, a simple relay channel is considered in which the channel coefficients are assumed to be circularly symmetric Gaussian distributed, and independent from each other. We then propose a new cooperation strategy to reduce SER. Our proposed scheme is based on this assumption that relay node can access to the channel information, therefore it can calculate the conditional symbol error probability of both DF and AF strategies. Then relay node selects the appropriate cooperation scheme and send its decision to destination. This algorithm is repeated when information of channels have an effective variety. Simulations verify this strategy can improve SER of cooperation network.

\section{REFERENCES}

1. Laneman J.N., G.W. Wornell, Distributed spacetime coded protocols for exploiting cooperative diversity in wireless networks, IEEE Transaction on Information Theory, 49, 2003, 2415-2525.

2. Laneman J.N., D.N.C. Tse, G. W. Wornell, Cooperative diversity in wireless networks: efficient protocols and outage behavior, IEEE Transaction on Information Theory, 50, 2004, 3062-3080.

3. Sendonaris A., E. Erkip, B. Aazhang, User cooperation diversity- Part I: system description, IEEE Transaction on Communications, 51, 2003, 1927-1938.

4. Sendonaris A., E. Erkip, B. Aazhang, User coop- 
eration diversity- Part II: implementation aspects and performance analysis, IEEE Transaction on Communications, 51, 2003, 1939-1948.

5. Hunter T.E., A. Nosratinia, Diversity through coded cooperation, IEEE Transaction on Wireless Communication, 5, 2006, 283-289.

6. Hunter T.E., A. Nosratinia, Performance analysis of coded cooperation diversity, IEEE International Conference on Communications, 4, 2003, 2688-2692.

7. Janani M., A. Hedayat, T. E. Hunter, A. Nosratinia, Coded cooperation in wireless communications: space-time transmission and iterative decoding, IEEE Transactions on Signal Processing, 52, 2004, $362-371$.

8. Stefanov A., E. Erkip, Cooperative coding for wireless networks, IEEE Transaction on Communications, 52, 2004, 1470-1476.

9. Su W., A.K. Sadek, K.J.R. Liu, Cooperative Communication Protocols in Wireless Networks: Performance Analysis and Optimum Power Allocation,
Springer Science + Business Media LLC, 2007.

10. Basiri Abarghouei M., A.M. Doost Hoseini, Cooperative communication with imperfect channel information: Performance analysis and optimum power allocation, Physical Communication, 4, 2011, 144-155.

11. Siriwongpairat W.P., T. Himsoon, W. Su, K. J. R. Liu, Optimum threshold-selection relaying for decode-and-forward cooperation protocol, in: Proceeding IEEE Wireless Communications and Networking Conference, Las Vegas, NV, April 2006, 1015-1020.

12. Brennan D.G., Linear diversity combining techniques, In Proceedings of the IEEE, 19 (2), 2003, 331-356.

13. Simon M.K., M. Alouini, A unified approach to the performance analysis of digital communication over generalized fading channels, in: Proceedings of the IEEE, 86 (9), 1998, 1860-1877.

14. Proakis J.G., Digital communications, 4th ed., McGraw-Hill: New York, 2001. 